\title{
The deep squat test has limited clinical utility for diagnosing symptomatic femoral acetabular impingement syndrome (FAIS): A systematic review
}

Simon Wood ( $\sim$ simon.wood@ouh.nhs.uk)

Physiotherapy Research Unit, Nuffield Orthopaedic Centre

Meredith Newman

Physiotherapy Research Unit, Nuffield Orthopaedic Centre

Martha Batting

Physiotherapy Research Unit, Nuffield Orthopaedic Centre

Karen Barker

Physiotherapy Research Unit, Nuffield Orthopaedic Centre

\section{Research Article}

Keywords: Squat test, diagnosis, femoral acetabular impingement

Posted Date: February 16th, 2022

DOI: https://doi.org/10.21203/rs.3.rs-1279690/v1

License: (a) (i) This work is licensed under a Creative Commons Attribution 4.0 International License.

Read Full License 


\section{Abstract}

\section{Purpose:}

Passive pain provocation tests (PPPT) are commonly used to diagnose FAIS but do not reflect functional restrictions. The deep squat test (DST) is a functional, weight-bearing test, but the clinical utility of the DST is unclear.

\section{Objective:}

To examine the validity and reliability of the DST in patients with confirmed FAIS

\section{Method:}

A Systematic Review registered with PROSPERO (CRD42020199249).

Seven databases; CINAHL, Cochrane, Embase, Medline, PubMed, SportDiscus and Web of Science, were searched up until December 2021. Data was extracted independently, and the Quality Assessment of Diagnostic Accuracy Studies (QUADAS-2) tool was used to evaluate the risk of bias.

\section{Result:}

1137 abstracts were found; 1119 were excluded after screening and 18 underwent full test review, excluding a further 17 , with one meeting the search criteria. No studies reported the reliability of the DST. One study ( $\mathrm{n}=78$ hips) investigated criterion-based validity: pain on DST indicated a positive test and was compared with MRI and MRA measures of FAIS. QUADAS score demonstrated low risk of bias. They concluded the DST had a modest potential to identify those with FAIS, but low specificity suggested the test may yield a high number of false positives.

\section{Conclusion:}

Physiotherapists should use the DST with caution. Single study evidence supports its use, but despite its common use, limited information is available about the DST and further investigation is required.

\section{Impact:}

Further studies are needed to examine validity and reliability, including evaluating the DST with higher alpha angles to determine whether diagnostic utility is improved.

\section{Introduction}

Femoral acetabular impingement syndrome (FAIS) is a common cause for hip and groin pain among adolescents and young adults(1). It is characterised by abnormal morphology of the proximal femur 
(CAM) and/or the acetabulum (PINCER) resulting in premature contact between the femoral head or neck and the acetabulum during hip flexion and internal rotation (2).

The overall prevalence of FAIS has been estimated to be between $10 \%$ to $15 \%$ of the population most of whom are age of 25 to 40 years (3) and are economically active. Therefore, FAIS leads to a significant cost burden for society as well as being individually debilitating (4).

The Warwick consensus meeting(4) in 2016 agreed upon three assessment criteria for diagnosing FAIS; clinical findings, symptoms, and radiographical findings. Positive findings from these three criteria aids in the diagnosis of FAIS.

Passive pain provocation tests (PPPT) are widely used in the clinical assessment of FAIS. The most frequently used are the Flexion, Abduction Internal Rotation test (FADIR) (5) and the Flexion Abduction and External Rotation distance test (FABERS) (6). The PPPT's rely on a clinician positioning the hip into a position where the CAM abuts the acetabulum to reproduce the patient's symptoms. However, Martin et al., (7)acknowledges that improperly performed tests could lead to false-positives or false-negative findings. Moreover, PPPT's do not replicate functional movements patterns that reflect the normal physiological load through the hip. Patients will often complain of pain and limitation in range of movement when the hip moves into a position of flexion and internal rotation. This position increases the potential impingement location e.g., during kicking sports (football, breaststroke etc) or sports requiring hyper-physiological movements (gymnastics, ballet etc) (4) but also in everyday activities such as squatting or sitting for prolonged periods (8) (4). Caliesch et al., (2020)(9) identified two functional tests: the Foot Position Angle Walk (FPAW)(5) and the maximal or Deep Squat Test (DST)(10), which are used in clinical settings to assist in the diagnosis of FAIS.

The DST places the hip joint under load in a position of maximal flexion and can initiate engagement of a CAM-type femoral deformity into the acetabular socket (10). It is a low cost, quick to administer tool, which could have clinical utility if proven to be reliable and a valid measure for diagnosing Cam FAI against a reference standard, but to date no review has examined the validity and reliability of the DST thus whether clinicians can confidently use the DST is unclear.

This systematic review aims to determine if the DST is a valid and reliable tool for the diagnosis of FAIS and whether it has clinical utility.

\section{Methods}

This review followed the Preferred Reporting Items for Systematic Reviews and Meta-Analyses (PRISMA) guidelines. The systematic review protocol was registered with Prospero, international prospective register for systematic review (CRD42020199249).

\section{Search strategy}


A comprehensive, reproducible literature search was carried out from January 1999 until December 2021 using the following data bases: Pubmed, EMBASE, Cochrane library, CINAHL, MEDLINE, Ovid, SPORTDiscus and Web of Science Core Collection. Search strategy example is included in Appendix 1. January 1999 was selected as the earliest retrieval record due to the first description of FAI (11). Boolean search terms, Medical Subject Headings (MeSH), synonyms and free text key words tailored to individual databases were used to search for studies in any language. See appendix 1 for an example search. Citations were tracked.

Two reviewers, SW and MB conducted the searches and screened titles and abstracts independently and blinded from each other's decision against a checklist of inclusion criteria. Included collated studies were then discussed between reviewers SW and MB and a third reviewer MN was referred to in the event of a disagreement. Full text articles were then identified and included and assessed for eligibility. The results of the searches were entered onto a pre-prepared, piloted standardised Excel (2013) spreadsheet.

Prior to final analyses a search re-run in December 2021 was conducted to include any previously unidentified papers.

\section{Eligibility criteria}

Primary studies were included if participants were aged between 15 and 60 years old and the index test was clearly described i.e., deep squat test (DST). Participants were also required to have a diagnosis of FAIS against a reference standard of either Magnetic Resonance Imaging (MRI) or Magnetic Resonance Arthrogram (MRA) and/or Computer tomography (CT) and/or X-ray. Studies conducted in any setting (hospital, laboratory, community etc) were eligible if they investigated measures of internal consistency or reliability of the squat test including test-re-test, inter-rater or intra-rater reliability and/or aspects of validity, construct, face or criterion. Studies were excluded if they involved participants younger than 15 years, or older than 60 years, or participants with other hip pathologies e.g., Osteoarthritis (OA), developmental hip joint abnormality, previous surgery, hip injection or conservative treatment in the preceding 3 months. Studies of the single leg squat test were excluded. No language restrictions were set.

\section{Data Extraction}

A data extraction tool was developed and pilot-tested by SW and MN with revisions made prior to use. Data extraction was performed by SW and verified by MB. Data extracted included: Country of origin, publication type, type of study, ethics, purpose of the study, study population and demographics, documentation of reference standard, diagnosis of FAIS, description of the index standard and measures of reliability and/or validity. 


\section{Quality assessment.}

The Quality Assessment of Diagnostic Accuracy Studies (QUADAS-2) was used to evaluate the risk of bias and applicability of primary diagnostic accuracy studies(12). The QUADAS-S risk of bias tool consists of four key domains: patient selection, index test, reference standard and flow and timing. Applicability of concerns covers 3 domains: patient selection, index standard test and reference standard test. The QUADAS-2 does not generate a summary "quality score", because of the known problems associated with such scores(13). A study is judged low risk of bias if all domains relating to bias or applicability are judged "low risk of bias" or "low concern regarding applicability". If a study is judged "high" or "unclear" on one or more domains, then it may be judged "at risk of bias" or as having "concerns regarding applicability".

One full text article met the eligibility and inclusion criteria for quality and risk of bias assessment. The selected article was independently assessed by both authors SW and MN using the QUADAS 2 tool and agreements discussed through consensus.

\section{Data Synthesis.}

Study characteristics were tabulated, and the risk of bias plotted. As only one study was identified, data synthesis was not possible. No sub-group analysis is planned.

\section{Results}

The search netted 1137 abstracts, following screening 18 studies were included for full-text review of which 17 were excluded as they did not meet the eligibility criteria. A search re-run in December 2021 did not identify any further articles. This left 1 study for data extraction. See Figure 1.

No reliability studies were identified investigating the DST in relation to FAIS. The single study identified investigated criterion validity (10) and therefore a risk of bias assessment was completed. No metaanalyses were possible. Details of the study characteristics are provided in Table1.

\section{Table 1}

Summary of Included study Table of description of main characteristics of study 


\begin{tabular}{|c|c|c|c|c|c|c|}
\hline $\begin{array}{l}\text { Author } \\
\text { Origin } \\
\text { Study Type }\end{array}$ & Participants & $\begin{array}{l}\text { Sensitivity } \\
(95 \% \mathrm{Cl})\end{array}$ & $\begin{array}{l}\text { Specificity } \\
(95 \% \mathrm{Cl})\end{array}$ & $\begin{array}{l}\text { LR +ve } \\
(95 \% \mathrm{Cl})\end{array}$ & $\begin{array}{l}\text { LR -ve } \\
(95 \% \mathrm{Cl})\end{array}$ & $\begin{array}{l}\text { QUADAS } \\
\text { RoB }\end{array}$ \\
\hline $\begin{array}{l}\text { Ayeni et al., } \\
(2014)(10)\end{array}$ & $\begin{array}{l}\cdot \mathrm{N}=76 \\
\bullet 39 \\
\text { Females }\end{array}$ & & & & & \\
\hline Canada & $\begin{array}{l}\text { - } 37 \text { Males } \\
\text { - All } \\
\text { symptomatic }\end{array}$ & $\begin{array}{l}75 \%(56- \\
88.5 \%)\end{array}$ & $\begin{array}{l}41 \%(27- \\
56.8 \%)\end{array}$ & $\begin{array}{l}1.3(0.9- \\
1.7)\end{array}$ & $\begin{array}{l}0.6(0.3- \\
1.2)\end{array}$ & Low \\
\hline $\begin{array}{l}\text { Pilot study of } \\
\text { validity }\end{array}$ & $\begin{array}{l}\text { - MRI or } \\
\text { MRA }\end{array}$ & & & & & \\
\hline
\end{tabular}

Cl= Confidence Intervals, LR=Likelihood Ratios, QUADAS RoB=Risk Of Bias, MRI= Magnetic Resonance imaging, MRA= Magnetic resonance arthrogram

The included study, Ayeni et al (2014)(10) demonstrated criterion-based validity and calculated the sensitivity and specificity of the deep squat test against the reference standard of imaging. They found the sensitivity of the DST (proportion of patients correctly classified with FAIS) to be $75 \%$ (95\% Cl 56.688.5). However, the specificity (proportion of patients correctly classified without FAIS) was $41 \%$ with (95\% $\mathrm{Cl} 27.0-56.8)$. The Likelihood ratio (LR)for a positive test was $1.3(95 \% \mathrm{Cl} 0.9-1.7)$ which means that a $30 \%$ pre-test probability is improved to $36 \%$ following a positive squat test. The LR for a negative test $1.3(95 \% \mathrm{Cl} 0.9-1.7)$ means that pre-test probability is improved to $20 \%$ with a negative squat test.

Ayeni et al., study was judged as 'at risk of bias' with 'Flow and timing' being unclear. Time frames between the reference standard being performed and the index test were not stated. There were no concerns for applicability (Table 2)

Table of result of the QUADAS-2 ROB Tool

\section{Table 2}

Results of the Quality Assessment for Diagnostic Accuracy Studies (QUADAS-2) Risk of Bias tool 


\begin{tabular}{|c|c|c|c|c|c|c|}
\hline \multicolumn{4}{|c|}{ Risk of Bias } & \multicolumn{3}{c|}{ Applicability concerns } \\
\hline $\begin{array}{c}\text { Patient } \\
\text { Selection }\end{array}$ & Index test & $\begin{array}{c}\text { Reference } \\
\text { standard }\end{array}$ & $\begin{array}{c}\text { Flow and } \\
\text { timing } \\
\text { Selection } \\
\text { standard } \\
\text { test }\end{array}$ & $\begin{array}{c}\text { Patient } \\
\text { Seference }\end{array}$ \\
\hline & - & - & 2 & & \\
\hline
\end{tabular}

\section{Low Risk of Bias ? Unclear}

Whiting P.F, Rutjes AWS, Westwood M.E, Mallett, et al.,(2011) QUADS-2:A Revised Tool for the Quality Assessment of Diagnostic Accuracy Studies. Annals of Interna Medicine; vol 155, issue 8

\section{Discussion}

The purpose of this study was to systematically review the literature for evidence on the validity and reliability of the DST when used to diagnose FAIS in order provide information about its clinical utility. Our search was systematic and thorough but revealed insufficient evidence about this commonly used test. There were no studies that investigated the reliability of the DST in a population of patients with FAIS, and only one study that evaluated criterion-based validity, reporting sensitivity and specificity.

\section{Reliability.}

The DST is used in other clinical settings and has been previously shown to have both validity and reliability in assessing strength in the lower limb for patients with cerebral palsy(CP) (14). The reliability of the DST from Ekens et al., (2020) (14) study cannot be generalised to patients with FAIS, insofar that the subjects were children with CP who were asked to perform two trials of 20 squats on the same day correlating squat pattern with leg strength. Furthermore, the reliability of the DST was judged on well executed squat rather than pain or restriction. Consequently, it is unknown if a patient with FAIS would consistently report pain and/or reduced squat height when tested at two separate time intervals or by different raters. Further work is required to standardise the DST for this hip pain population and test both its intra (test-re-test) and interrater reliability.

\section{Face Validity}

The face validity of the DST remains high. Squatting is frequently reported as a problem during daily life by patients with FAIS and is incorporated within validated, rigorously developed self-administered patient reported outcome measures, the iHOT33 and Hip Outcome Score (HOS). The HOS explores whether one 
can deep squat with 'no difficulty at all' to 'unable to perform' whereas the iHOT33 relates the problem of crouching or squatting to work based occupational activities.

These questions within the iHOT33 and HOS have been included because psychometric testing has identified that squatting is an important functional domain of the experience of hip pathologies like FAIS not captured by other measures.

\section{Construct validity}

The construct theorem of the DST postulates that during a deep squat, there is abutment of the CAM against the acetabulum resulting in symptoms of pain, stiffness, reduced range of squat depth or altered squat pattern. This is supported by laboratory studies.

However, Ayeni's study has showed that the sensitivity of the DST was $75 \%$ suggesting that $25 \%$ of participants were able to achieve a painless full squat who may have CAM morphology. Furthermore, the low specificity $(41 \%)$ suggests that this test may yield a high number of false positives, i.e., painful, or limited squat in the absence of FAIS.

This could be explained by laboratory-based studies using motion capture cameras which have investigated the performance of the DST in patients with confirmed FAIS who present with limited squat depth $((8,15,16)$. Bagwell et al.,(15) found that people with pain and stiffness due to CAM deformity had reduced femoral internal rotation moment and a relatively anteriorly tilted pelvis due to loss of the ability to posteriorly pelvic tilt at the approach of maximal squat, resulting in reduction in squat depth. The authors hypothesised that decreased posterior pelvic tilt would be expected to increase impingement between the femur and the acetabulum, during a task involving deep hip flexion.

Similarly, Lamontagne 2009(8) showed that participants with FAIS during maximal squat had less pelvic recline, and hypothesised that it is reasonable to assume that cam FAl causes a general decrease in pelvic sagittal ROM predisposing the hip to premature contact between the proximal femur and the acetabular rim in movements requiring deep hip flexion. However, in contrast, Mullins et al., (2018)(17) reported no difference in squat depth in participants with confirmed FAIS compared to controls, although within the FAl group, $56 \%$ ( $n=30$, of 54 subjects with 78 hips included) reported anterior groin pain and a further $18 \%(n=4)$ reported stiffness in the hip. Diamond et al., (18) also found that squat depth was equal between controls but the symptomatic FAIS group during an unconstrained squat had a slower descent speed on squatting and the ipsilateral side of the pelvis did not descend as far. This movement pattern is believed to reduce the demand for hip flexion. Greater hip adduction was also found to be a significant finding by Kumar et al., (2014)(19) and greater internal rotation moment during the DST in participants with FAIS who had a mean alpha angle of 74 deg, suggesting that the hip joint moves into an impingement position during the DST. 
In summary, changes in squat depth therefore could be explained by a number of biomechanical anomalies such as reduced sagittal pelvic posterior rotation, hip adduction or greater internal rotation moment. Potentially, the CAM morphology could be causing abutment with the acetabulum or the lack of sagittal posterior rotation of the pelvis causing abutment of the acetabulum with the CAM. A more posteriorly tilted pelvis may explain why some patients with FAIS can perform the DST without pain/reduced range.

Moreover, it is unlikely whether a clinician can detect the nuances of altered squat biomechanics described in the laboratory models within a clinical setting. However, it seems reasonable to determine whether a number of factors during squat performance such as: pain, depth, pelvic rotation and pelvis asymmetry explain the variability in the DST.

To further add to the difficulties of using the DST for diagnosing FAIS, Ayeni's et al., study highlighted multi-dependent variables within the patient group that could explain the further discrepancy in the squat performance. For example, patients with/without FAIS also had other co-pathologies including labral tear, gluteus Medius tendinopathy, cartilage damage, etc. This may introduce bias validity in the assessment of the DST for diagnosing FAl insofar that in a real-world clinical setting, co-existing hip pathologies will be abundant thus possibly limiting the clinical utility of the test to a small number of patients without coexisting hip pathology.

\section{Quality assessment}

Ayeni's et al (10)study was graded at risk of bias with concerns with 'flow and timing'. This is in contrast to (9) who judged Ayeni's study as low risk of bias. Flow and timing relate to the time frames between the reference standard (MRI/A) being performed and the index test (DST) given. This was not clear in Ayeni's study. This may mean that the MRI findings may not be a true indication of the participants hip at the time of the index test, if the recruitment of the participant were based on outdated MRI/A's images. This could result in change over time in the configuration of the alpha angle from baseline, and/or progression in co-morbidities which could hypothetically influence the DST performance. Therefore, a progression of co-morbidities could produce a false positive or a false negative result, thus introducing bias into the results of the DST. It is not known how changes in MRI/A appearances influence the DST therefore the relevance of timing remains unclear

\section{Alpha angle}

The alpha angle measures the extent to which the femoral head deviates from spherical (20) and is a radiological measurement proposed for the detection of cam morphology in patients with FAIS but there is no one value that can define cam morphology(21). Cam morphology is highly prevalent in the general population and can be either symptomatic and asymptomatic. Agricola et al.,(20) found that an alpha angle $<60$ deg could not discriminate between hips with and without CAM deformity but agreed with two 
previous studies that an alpha angle of $>60$ deg has been shown to discriminate between symptomatic and asymptomatic CAM deformities. A more recent study by van Klij et al., (22) supported a diagnostic alpha angle cut off value of $\geq 60$ deg but recommended further research to evaluate whether this threshold is applicable to all imaging modalities prior to acceptance. Another study also proposed a radiographic alpha angle of $\geq 60$ deg suggesting a cam-lesion and a radiographic alpha angle of $78 \mathrm{deg}$ indicating the presence of a non-gender specific presence of pathological cam-lesion thought to lead to end-stage OA (20). Ayeni's cut off alpha angle was $55 \mathrm{deg}$ (without documentation of the mean alpha angle or standard deviation), therefore it may be reasonable to suggest that the findings regarding the sensitivity of the DST may have been negatively influenced by inclusion of patients with non-pathological CAM. If the study were repeated with a higher alpha angle cut off point i.e., $\geq 60$ deg, the sensitivity and specificity of the DST and hence its diagnostic utility might improve. Furthermore, when using the alpha angle to categorise a CAM morphology, according to Dijkstra et al.,(21) there is no consensus on imaging outcomes specific to cam morphology, or when anatomy flips to pathology. To date, there is no agreement on a diagnostic alpha angle cut-off value. Thus, further studies are needed to investigate the criterion validity of the DST with a range of alpha angles, including $\geq 60$ deg and $\geq 70$ deg.

\section{Squat test standardisation}

The DST offers a novel screening task that may prove challenging to some patients. Variability in DST may be a product of lack of familiarity with the task, movement impairment due to pathology and/or lack of standardisation of the task. There is evidence that changing factors such as stance width alters muscle activation, joint kinematics and joint forces in a squat position (23) Furthermore, changing arm position and allowing self-selection of foot position during DST in asymptomatic participants alters performance McMillian et al., (2016) (24) and improved the 3 functional movement scores used to assess the movement pattern: upper torso parallel to tibia or vertical, femur below horizontal, knees aligned over feet.

Laboratory kinematic and EMG studies of the DST show no agreement on a standard squat test position for FAIS. Of 9 laboratory studies investigating the DST with confirmed FAIS, 3 encouraged participants to squat to a depth of $1 / 3$ of tibial height $(8,15,17)$, 4 studies encouraged participants to squat as deep as possible(10,16, 18, 25), 2 studies required the participants to squat to a self-selected depth or pace(18, 26 ) and one study squatting to $25 \%$ of body height (19). One study included placement of a 30 degree wedge under the participants heals to encourage deep hip flexion and unrestricted by ankle dorsiflexion during constrained and unconstrained squatting. Furthermore, control of heel position varied between studies, from heel off $(n=2)$, no control $(n=3)$, maintain heel contact $(n=3)$, during the DST.

Rigid standardisation of the squat test controlling for verbal cues, foot position, stance width and arm position could likely change the squat movement pattern which individuals would naturally adopt. In everyday life, one does not check these elements prior to squatting thus rigid standardisation of the DST could artificially change a natural movement pattern. In contrast, non-standardisation of the DST makes 
the measurements of reliability and validity challenging with regards to a lack of consistency between raters. Therefore, there must be a compromise so that the DST is repeatable but reflects normal movement patterns. Elements to standardise the DST for example could be footwear (no shoes), arms by the side in an indoor environment and instructed to squat as low as possible. Objective standardisation could include measures of symptoms, e.g., pain and/or stiffness and measures of depth of squat such as a grid or ruler attached to a wall. Standardisation of observation to be recorded on a form to observe quality of the DST, i.e., symmetry, pain and range or depth of DST. Thus, a semi standardised DST can be used for a consistent DST for reliability without changing the movement pattern. Furthermore, a lack of practice and/or experience in the movement pattern in the DST may introduce test bias affecting the performance and degrade the DST and directly affecting its validity and reliability. Arguably a pre-test DST may be required to familiarise the participants with the motor task, but would this affect the DST pattern through a learning effect? Therefor the question arises; how much does one standardise the DST without affecting its validity or reliability?

\section{DST combined with PPPT's}

The two most commonly used passive pain provocation tests; Flexion, abduction and external rotation test (FABERS) and the flexion, adduction, internal rotation test (FADIRS) are used to assist in the diagnosis of FAIS. A recent systematic review (9) investigated sensitivity and specificity for these two tests and found the FADIRS test was $0.96(\mathrm{Cl} 0.91-0.99)$ and $0.11(\mathrm{Cl} 0.06-0.20)$ and the FABERS $0.85(\mathrm{Cl}$ $0.79-0.90)$ and 0.38 ( $\mathrm{Cl} 0.33-0.42)$ respectively. The likelihood ratios for these two tests were poor: FADIRS, LR+ 1.079 (0.99-1.17), LR- 0.364 (0.12-1.08) and FABERS LR+ 1.36 (1.34 to 1.50), LR-0.41 (0.280.59). In a clinical setting these two commonly used tests in combination with the DST would be quickly and easily administered. However, what is not known is whether the diagnostic utility of the DST i.e. ruling in FAIS, is improved when used in conjunction with FABERS and FADIRS or whether the combination of these three tests improve the post test probability for diagnosing FAIS. Further investigation of these three tests warrants further research to determine whether these tests add to the diagnosis of FAIS in a clinical setting.

\section{Conclusion}

There is limited evidence to support the use of the DST in the diagnosis of FAIS in a clinical environment. Laboratory studies provide some preliminary evidence of construct validity. The DST has reasonable face validity but no studies to date demonstrate its reliability. Furthermore, confounding influences may affect the DST such as non-standardisation of the DST, concurrent hip comorbidities and other biomechanical considerations. A cautious approach to using the DST in the diagnosis of FAIS should be advised due to the risk of false positives or negatives. Furthermore, used in combination with other commonly used tests, the diagnostic utility remains unclear. 
Finally, the lack of agreement of classifying pathological CAM morphology from an agreed alpha angle remains elusive and therefor the DST may become a more valid and reliable tool as the alpha angle cut off point is raised.

\section{Limitations}

The most notable limitation to this review were the lack of articles retrieved from the search, with only one relevant paper and therefore limited evidence to support the use of the DST in the diagnosis of FAI. Thus, reliability and validity have not been robustly challenged. Furthermore, spectrum bias may exist because Ayenis et al., study only selected individuals with hip pain from one clinic that were heterogenous and may explain the variation in the DST.

\section{Recommendation}

Further research is required to investigate the reliability of the DST to determine if it has clinical utility, and whether clinicians have the observational skills to identify pelvic/hip movement patterns that are thought to affect the DST. Does combining DST with the two most commonly used clinical tests increase diagnostic utility? Finally, would an increase in the alpha angle improve the sensitivity and specificity of the DST?

\section{Abbreviations}

$\mathrm{CP}$

CT

DST

EMG

FABERS

FADIRS

FAI

FAIS

FPAW

HOS

iHOT33 
OA

$\mathrm{MB}$

$\mathrm{MeSH}$

MN

$\mathrm{MRI} / \mathrm{A}$

PPPT

PRISMA

QUADAS-2 ROB

ROM

SW

Declarations

Ethics approval:

Not Applicable

\section{Consent for publication:}

Yes

\section{Availability of data and materials:}

All data available from the following databases: Pubmed, EMBASE, Cochrane library, CINAHL, MEDLINE, Ovid, SPORTDiscus and Web of Science Core Collection. search databases form text

\section{Competing interests:}

Nil

\section{Source(s) of Support/Funding:}


This work was completed as part of a National Institutes of Health Research Pre-Doctoral Academic Fellowship [HFR03660].

\section{Authors contribution:}

Simon Wood: Study conception, design, data acquisition and interpretation, drafting the article and revising manuscript and final approval

Meredith Newman: Study conception, interpretation, revising manuscript, final approval

Martha Batting: Data acquisition, data analysis and interpretation, revising manuscript

Karen Barker: Interpretation, revising manuscript, final approval

\section{Acknowledgements:}

I am grateful to Liz Callow, librarian at the Bodleian Library, for advice/help on compiling a literature search strategy

\section{References}

1. Pennock AT, Bomar JD, Johnson KP, Randich K, Upasani VV. Nonoperative Management of Femoroacetabular Impingement: A Prospective Study. Am J Sports Med. 2018;46(14):3415-22.

2. Ganz R, Parvizi J, Beck M, Leunig M, Notzli H, Siebenrock KA. Femoroacetabular impingement: a cause for osteoarthritis of the hip. Clin Orthop Relat Res. 2003(417):112-20.

3. Wylie JD, Peters CL, Aoki SK. Natural History of Structural Hip Abnormalities and the Potential for Hip Preservation. J Am Acad Orthop Surg. 2018;26(15):515-25.

4. Griffin DR, Dickenson EJ, O'Donnell J, Agricola R, Awan T, Beck M, et al. The Warwick Agreement on femoroacetabular impingement syndrome (FAl syndrome): an international consensus statement. $\mathrm{Br}$ J Sports Med. 2016;50(19):1169-76.

5. Ranawat AS, Gaudiani MA, Slullitel PA, Satalich J, Rebolledo BJ. Foot Progression Angle Walking Test: A Dynamic Diagnostic Assessment for Femoroacetabular Impingement and Hip Instability. Orthop J Sports Med. 2017;5(1):2325967116679641.

6. Maslowski E, Sullivan W, Forster Harwood J, Gonzalez P, Kaufman M, Vidal A, et al. The diagnostic validity of hip provocation maneuvers to detect intra-articular hip pathology. PM R. 2010;2(3):174-81.

7. Martin HD, Kelly BT, Leunig M, Philippon MJ, Clohisy JC, Martin RL, et al. The pattern and technique in the clinical evaluation of the adult hip: the common physical examination tests of hip specialists. Arthroscopy. 2010;26(2):161-72. 
8. Lamontagne M, Kennedy MJ, Beaule PE. The effect of cam FAl on hip and pelvic motion during maximum squat. Clin Orthop Relat Res. 2009;467(3):645-50.

9. Caliesch R, Sattelmayer M, Reichenbach S, Zwahlen M, Hilfiker R. Diagnostic accuracy of clinical tests for cam or pincer morphology in individuals with suspected FAl syndrome: a systematic review. BMJ Open Sport Exerc Med. 2020;6(1):e000772.

10. Ayeni O, Chu R, Hetaimish B, Nur L, Simunovic N, Farrokhyar F, et al. A painful squat test provides limited diagnostic utility in CAM-type femoroacetabular impingement. Knee Surg Sports Traumatol Arthrosc. 2014;22(4):806-11.

11. Myers SR, Eijer H, Ganz R. Anterior femoroacetabular impingement after periacetabular osteotomy. Clin Orthop Relat Res. 1999(363):93-9.

12. Whiting PF, Rutjes AW, Westwood ME, Mallett S, Deeks JJ, Reitsma JB, et al. QUADAS-2: a revised tool for the quality assessment of diagnostic accuracy studies. Ann Intern Med. 2011;155(8):529-36.

13. Whiting P, Harbord R, Kleijnen J. No role for quality scores in systematic reviews of diagnostic accuracy studies. BMC Med Res Methodol. 2005;5:19.

14. Eken MM, Dallmeijer AJ, Buizer Al, Hogervorst S, van Hutten K, Piening M, et al. Intraobserver Reliability and Construct Validity of the Squat Test in Children With Cerebral Palsy. Pediatr Phys Ther. 2020;32(4):399-403.

15. Bagwell JJ, Snibbe J, Gerhardt M, Powers CM. Hip kinematics and kinetics in persons with and without cam femoroacetabular impingement during a deep squat task. Clin Biomech (Bristol, Avon). 2016;31:87-92.

16. Catelli DS, Kowalski E, Beaule PE, Smit K, Lamontagne M. Asymptomatic Participants With a Femoroacetabular Deformity Demonstrate Stronger Hip Extensors and Greater Pelvis Mobility During the Deep Squat Task. Orthop J Sports Med. 2018;6(7):2325967118782484.

17. Mullins K, Hanlon M, Carton P. Differences in Athletic Performance Between Sportsmen With Symptomatic Femoroacetabular Impingement and Healthy Controls. Clin J Sport Med. 2018;28(4):370-6.

18. Diamond LE, Bennell KL, Wrigley TV, Hinman RS, O'Donnell J, Hodges PW. Squatting Biomechanics in Individuals with Symptomatic Femoroacetabular Impingement. Med Sci Sports Exerc. 2017;49(8):1520-9.

19. Kumar D, Dillon A, Nardo L, Link TM, Majumdar S, Souza RB. Differences in the association of hip cartilage lesions and cam-type femoroacetabular impingement with movement patterns: a preliminary study. PM R. 2014;6(8):681-9.

20. Agricola R, Waarsing JH, Thomas GE, Carr AJ, Reijman M, Bierma-Zeinstra SM, et al. Cam impingement: defining the presence of a cam deformity by the alpha angle: data from the CHECK cohort and Chingford cohort. Osteoarthritis Cartilage. 2014;22(2):218-25.

21. Dijkstra HP, Ardern CL, Serner A, Mosler AB, Weir A, Roberts NW, et al. Primary cam morphology; bump, burden or bog-standard? A concept analysis. Br J Sports Med. 2021. 
22. van Klij P, Reiman MP, Waarsing JH, Reijman M, Bramer WM, Verhaar JAN, et al. Classifying Cam Morphology by the Alpha Angle: A Systematic Review on Threshold Values. Orthop J Sports Med. 2020;8(8):2325967120938312.

23. Escamilla RF, Fleisig GS, Lowry TM, Barrentine SW, Andrews JR. A three-dimensional biomechanical analysis of the squat during varying stance widths. Med Sci Sports Exerc. 2001;33(6):984-98.

24. McMillian DJ, Rynders ZG, Trudeau TR. Modifying the Functional Movement Screen Deep Squat Test: The Effect of Foot and Arm Positional Variations. J Strength Cond Res. 2016;30(4):973-9.

25. Diamond LE, van den Hoorn W, Bennell KL, Wrigley TV, Hinman RS, O'Donnell J, et al. Deep hip muscle activation during squatting in femoroacetabular impingement syndrome. Clin Biomech (Bristol, Avon). 2019;69:141-7.

26. Rutherford DJ, Moreside J, Wong I. Differences in Hip Joint Biomechanics and Muscle Activation in Individuals With Femoroacetabular Impingement Compared With Healthy, Asymptomatic Individuals: Is Level-Ground Gait Analysis Enough? Orthop J Sports Med. 2018;6(5):2325967118769829.

\section{Figures}

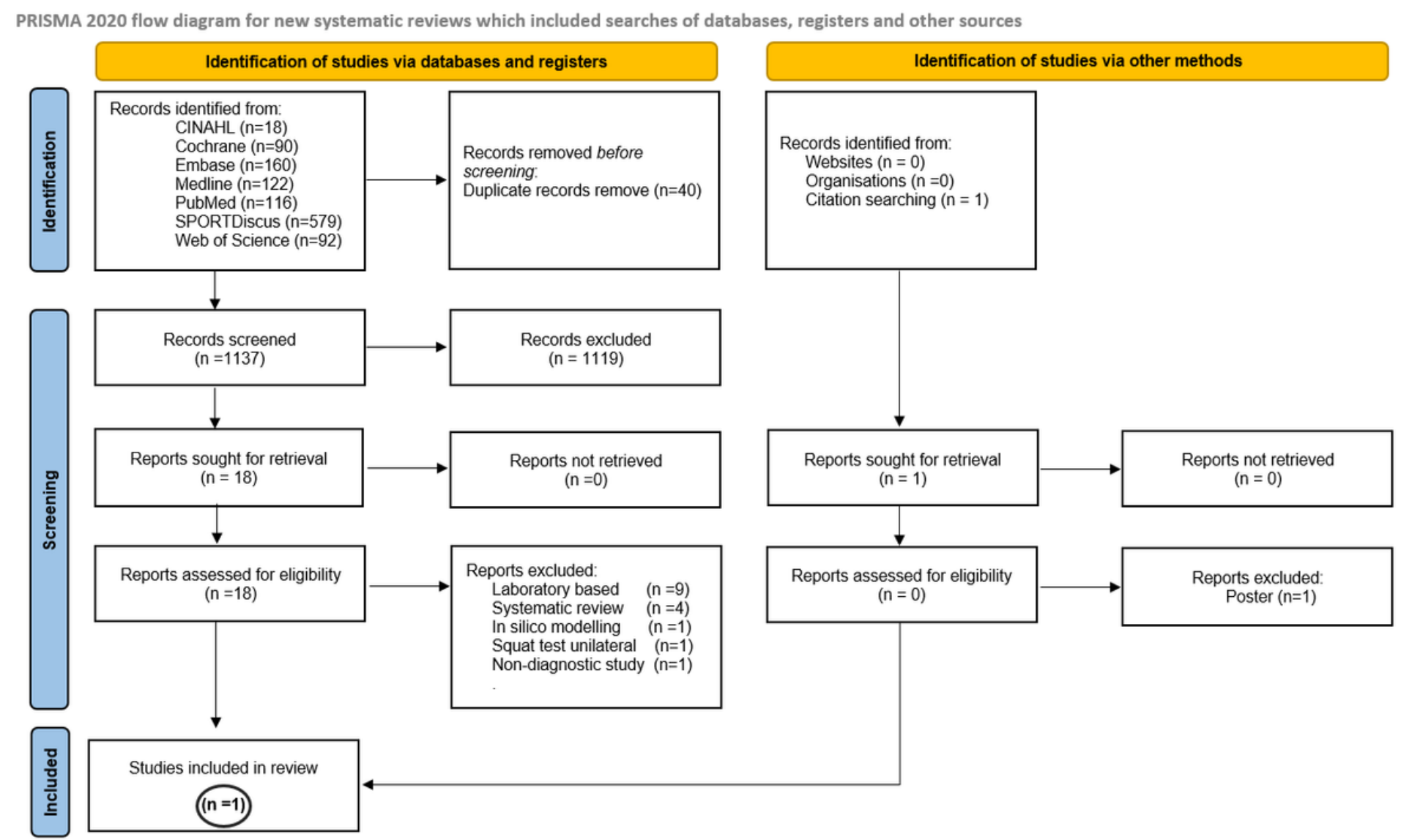

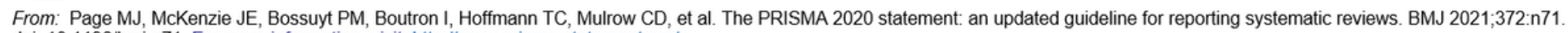
doi: 10.1136/bmj.n71. For more information, visit: http://www.prisma-statement.org/

\section{Figure 1}


PRISMA study flow diagram

Page 17/17 